\title{
A New Solution for The Enzymatic Glucose Fuel Cell Model with Morrison Equation via Haar Wavelet Collocation Method
}

\author{
Kuntida Kawinwit, Akapak Charoenloedmongkhon ${ }^{*}$, Sanoe Koonprasert \\ Department of Mathematics, Faculty of Applied Science King Mongkut's University of Technology North Bangkok, \\ Bangkok 10800, Thailand
}

\begin{abstract}
Received December 24, 2020; Revised March 15, 2021; Accepted March 26, 2021
Cite This Paper in the following Citation Styles

(a): [1] Kuntida Kawinwit, Akapak Charoenloedmongkhon, Sanoe Koonprasert, "A New Solution for The Enzymatic Glucose Fuel Cell Model with Morrison Equation via Haar Wavelet Collocation Method," Mathematics and Statistics, Vol.9, No.2, pp. 188-198, 2021. DOI: 10.13189/ms.2021.090214.

(b): Kuntida Kawinwit, Akapak Charoenloedmongkhon, Sanoe Koonprasert, (2021). A New Solution for The Enzymatic Glucose Fuel Cell Model with Morrison Equation via Haar Wavelet Collocation Method. Mathematics and Statistics, 9(2), 188-198. DOI: 10.13189/ms.2021.090214.
\end{abstract}

Copyright $@ 2021$ by authors, all rights reserved. Authors agree that this article remains permanently open access under the terms of the Creative Commons Attribution License 4.0 International License

\begin{abstract}
Integral equations are essential tools in various areas of applied mathematics. A computational approach to solving an integral equation is important in scientific research. The Haar wavelet collocation method (HWCM) with operational matrices of integration is one famous method which has been applied to solve systems of linear integral equations. In this paper, an approximated analytical method based on the Haar wavelet collocation method is applied to the system of diffusion convection partial differential equations with initial and boundary conditions. This system determines the enzymatic glucose fuel cell with the chemical reaction rate of the Morrison equation. The enzymatic glucose fuel cell model describes the concentration of glucose and hydrogen ion that can be converted into energy. During the process, the model reduces to the linear integral equation system including computational Haar matrices. The computational Haar matrices can be computed by HWCM coding in the Maple program. Illustrated examples are provided to demonstrate the preciseness and effectiveness of the proposed method. The results are shown as numerical solutions of glucose and hydrogen ion.
\end{abstract}

Keywords Morrison equation, Haar Wavelet Collocation Method, Partial Differential Equation, Enzymatic Glucose Fuel Cell

\section{Introduction}

Nonlinear differential equations (NDEs) play significant roles in various areas of applied sciences, for example, mathematical biology, fluid mechanics, chemical kinetics, temperature distribution, reaction-diffusion system, industrial process, and plasma physics.

Many researchers have now obtained several types of methods to solve approximate analytical solutions of partial differential equations (PDEs) including the Chebyshev wavelet collocation method (CWCM) [9], the Haar wavelet collocation method (HWCM) [6], the Legendre wavelet collocation method (LWCM) [18], the Laplace Adomian decomposition method (LADM) [19], the Homotopy perturbation method (HPM) [22] and the variational iteration method (VIM) [5], etc.

In 1997, Chen and Hsiao [3] first developed the Haar wavelet method via an operational matrix of integration related to the Haar function vector. In 2005, Lepik [24] solved the higher order with nonlinear ordinary differential and evolution equations using the Haar wavelet method. Recently, the Haar wavelet method is used as a mathematical tool for reaction-diffusion partial differential equations in many applications [8] such as chemical reactions, biological chemistry and fluid mechanics.

In biology and chemistry systems, PDEs play a role in various processes in the structure of human beings, such as the carbon dioxide $\left(\mathrm{CO}_{2}\right)$ and oxygen $\left(\mathrm{O}_{2}\right)$ diffusions in the bloodstream [4], oxygen diffusion in absorbing tissue [13], enzyme kinetics reaction mechanisms [1], enzymatic glucose fuel cell [23], etc. Various types of enzymes play important roles in cells to help speed up numerous vital reactions. Enzymes are specific biocatalysts, meaning that they are specific to one type 
of reaction and a small group of reactants called substrates. Kinetics is the study of reaction rates, where the rate of the reaction means the rate of product formation. Enzyme kinetics has a long history, as it is one of the earliest areas where mathematics was utilized to understand biological phenomena. In addition, various structures for the $\mathrm{pH}$, the concentrations of reaction, the temperature values of reaction media and mass transport effects are applied in enzyme kinetics.

The chemical reaction of enzymatic glucose fuel cell between enzyme and glucose is shown in Figure 1.

$$
E+C_{G} \frac{K_{o n}}{\sum_{o f f}} \sum E C_{G} \stackrel{K_{c a t}}{>E}+P
$$

Figure 1. Schematic diagram of the chemical reaction between enzyme and glucose.

Here, $E$ is the enzyme, $C_{G}$ is the glucose (substrate), $E C_{G}$ is the enzyme-glucose complex and $P$ is the product. In Figure 1 , the Michaelis Menten equation [16] is one of the best-known reaction rates when $K_{M}>\left[E_{T}\right]$. The reaction rate $f\left(\left[C_{G}\right]\right)$ is given by

$$
f\left(\left[C_{G}\right]\right)=\frac{V_{\max }\left[C_{G}\right]}{K_{M}+\left[C_{G}\right]} .
$$

On the other hand, when $K_{M}<\left[E_{T}\right]$, the appropriated reaction rate can be provided by the Morrison equation (Quadratic Velocity equation) [11] with the reaction rate $f\left(\left[C_{G}\right]\right)$ as

$$
f\left(\left[C_{G}\right]\right)=V_{\max } \frac{\left(a+\left[C_{G}\right]\right)-\sqrt{\left(a+\left[C_{G}\right]\right)^{2}-4\left[E_{T}\right]\left[C_{G}\right]}}{2[E]},
$$

where $a=[E]+K_{M},\left[E_{T}\right]=[E], K_{M}=\frac{K_{o f f}+K_{c a t}}{K_{o n}}$. Many researchers are interested in the performance of enzymatic glucose fuel cells. Scott Barton [21] studied enzymatic glucose fuel cells in various types of models. Rajendran [15] solved the approximate analytical solution for a mono enzymatic biosensor with the Michaelis Menten equation via the Homotopy perturbation method. Malinidevi [17] studied the reaction and diffusion of enzymes immobilized in an artificial membrane with a mathematical model. The enzymatic glucose fuel cell uses glucose as a fuel to generate electrical energy and enzymes as a biocatalyst to change chemical energy into electrical energy [14]. The anodic reaction is given by

$$
\mathrm{C}_{6} \mathrm{H}_{12} \mathrm{O}_{6}+\mathrm{H}_{2} \mathrm{O} \rightarrow \mathrm{C}_{6} \mathrm{H}_{12} \mathrm{O}_{7}+2 \mathrm{e}^{-}+2 \mathrm{H}^{+},
$$

and the cathodic reaction is given by

$$
\frac{1}{2} \mathrm{O}_{2}+2 \mathrm{H}^{+}+2 \mathrm{e}^{-} \rightarrow \mathrm{H}_{2} \mathrm{O} .
$$

The diagram in Figure 2 assumes one-dimensional diffusion transport equations of glucose $\left(\mathrm{C}_{6} \mathrm{H}_{12} \mathrm{O}_{6}\right)$ and hydrogen ion $\left(\mathrm{H}^{+}\right)$across a fuel cell. The glucose moves in from the anode, which has oxidation, to the cathode which has reduction. In the enzyme layer, a reaction occurs in which the glucose and enzyme become gluconic acid $\left(\mathrm{C}_{6} \mathrm{H}_{12} \mathrm{O}_{7}\right)$, hydrogen ion and

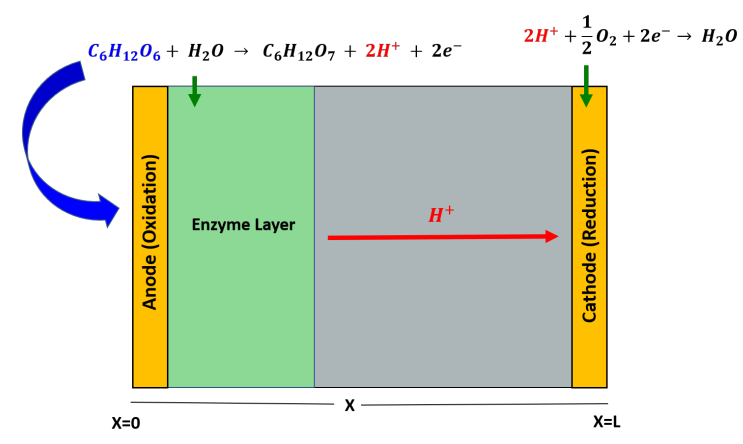

Figure 2. Schematic diagram of the reaction rate of enzymatic glucose fuel cell.

electron ion. Then the hydrogen ion reacts with oxygen in cell wall (cathode), after which it becomes water $\left(\mathrm{H}_{2} \mathrm{O}\right)$.

The glucose concentration $\left(\left[C_{G}\right]\right)$ and the hydrogen ion concentration $\left(\left[C_{H^{+}}\right]\right)$can be determined by the model of the transport equation with the reaction rates of the Morrison equation as

$$
\begin{aligned}
\frac{\partial\left[C_{G}\right]}{\partial t}= & D_{G} \frac{\partial^{2}\left[C_{G}\right]}{\partial x^{2}} \\
& -\frac{V_{\max }}{2[E]}\left[\left(a+\left[C_{G}\right]\right)-\sqrt{\left(a+\left[C_{G}\right]\right)^{2}-4[E]\left[C_{G}\right]}\right], \\
\frac{\partial\left[C_{H^{+}}\right]}{\partial t}= & D_{H^{+}} \frac{\partial^{2}\left[C_{H^{+}}\right]}{\partial x^{2}}-y D_{H^{+}} \frac{\triangle v^{*}}{L} \frac{\partial\left[C_{H^{+}}\right]}{\partial x} \\
& +\frac{V_{\max }}{[E]}\left[\left(a+\left[C_{G}\right]\right)-\sqrt{\left(a+\left[C_{G}\right]\right)^{2}-4[E]\left[C_{G}\right]}\right],
\end{aligned}
$$

where $0<x<L, t>0$. The initial conditions are

$$
\begin{aligned}
{\left[C_{G}\right](x, 0) } & =1, \\
{\left[C_{H^{+}}\right](x, 0) } & =0,
\end{aligned}
$$

and the boundary conditions are

$$
\begin{aligned}
{\left[C_{G}\right](0, t) } & =1, \\
\frac{\partial\left[C_{G}\right]}{\partial x}(L, t) & =0, \\
{\left[C_{H^{+}}\right](0, t) } & =0, \\
\frac{\partial\left[C_{H^{+}}\right]}{\partial x}(L, t) & =0 .
\end{aligned}
$$

In this paper, we introduce the Haar wavelet collocation method (HWCM) and apply this method to find an approximate analytical solution of the enzymatic glucose fuel cell with the Morrison equation model. Coding of HWCM in the Maple program is developed to show some numerical results of the model, which can describe levels of glucose $\left(C_{G}\right)$ and hydrogen ion concentration $\left(C_{H^{+}}\right)$Eq.(5).

\section{The Haar Wavelet Collocation Method}

The Haar wavelet is known as the simplest example of an orthogonal wavelet, which is defined by a square wave function 


\begin{tabular}{cl}
\hline Symbols & Parameters \\
\hline$\left[C_{G}\right]$ & Glucose concentration \\
{$\left[C_{H^{+}}\right]$} & Hydrogen ion concentration \\
$D_{G}$ & Diffusion coefficient of glucose \\
$D_{H^{+}}$ & Diffusion coefficient of hydrogen ion \\
{$[E]$} & Enzyme concentration at enzyme layer \\
{$\left[E_{T}\right]$} & Total enzyme concentration \\
$K_{c a t}$ & Kinetic enzyme reaction rate constant \\
& for glucose oxidation \\
$V_{\text {max }}$ & The maximum velocity achieved by the \\
& system at maximum (saturating) glucose \\
& concentration $V_{\text {max }}=K_{\text {cat }}[E]$ \\
$K_{M}$ & Michaelis Menten or Morrison constant \\
$\triangle v^{*}$ & Dimensionless voltage drop in the cell, \\
$L$ & Distance between anode and cathode, \\
$y$ & Stoichiometric coefficient, \\
$x$ & Coordinate direction normal to the anode, \\
$t$ & Time.
\end{tabular}

on the unit interval $[0,1]$ as

$$
h_{1}(x)= \begin{cases}1, & x \in[0,1) \\ 0, & \text { otherwise }\end{cases}
$$

and the scaling functions, for $i=2,3, \ldots$,

$$
h_{i}(x)=\left\{\begin{array}{cc}
1, & x \in\left[\alpha_{1}, \alpha_{2}\right), \\
-1, & x \in\left[\alpha_{2}, \alpha_{3}\right), \\
0, & \text { otherwise },
\end{array}\right.
$$

where $\alpha_{1}=\frac{k}{m}, \alpha_{2}=\frac{k+0.5}{m}, \alpha_{3}=\frac{k+1}{m}$ and the dilations integer $m=2^{j}, j=0,1, \ldots, J$ indicates the level of the wavelet with the maximal level of resolution $J$ and $k=0,1, \ldots, m-1$ is the translation parameter as shown in the following Figure 3.

The index $i$ in Eq. (9) is expressed by the integers $m$ and $k$ as $i=m+k+1$. It is obvious that the minimal value of $i$ is $i=2$ when $m=1, k=0$ and the maximal value of $i$ is $i=2^{J+1}$ when $m=2^{J}, k=2^{J}-1$.

The Haar wavelet functions satisfy the following properties:

$$
\int_{0}^{1} h_{i}(x) d x= \begin{cases}1, & \text { if } i=1, \\ 0, & \text { if } i=2,3, \ldots,\end{cases}
$$

and the orthogonal property for $i, l=1,2, \ldots$

$$
\int_{0}^{1} h_{i}(x) h_{l}(x) d x= \begin{cases}2^{-j}, & i=l=2^{j}+k \\ 0, & i \neq l\end{cases}
$$

The Haar wavelet function can construct a very good transform basis which is represented by any square integrable function, $u(x)$, on $(-\infty, \infty)$, i.e. $\int_{-\infty}^{\infty} u^{2}(x) d x<\infty$. Therefore, any function $u(x)$ can be expressed in terms of an infinite sum of the Haar wavelets as follows:

$$
u(x)=\sum_{i=1}^{\infty} a_{i} h_{i}(x), \quad x \in[0,1),
$$
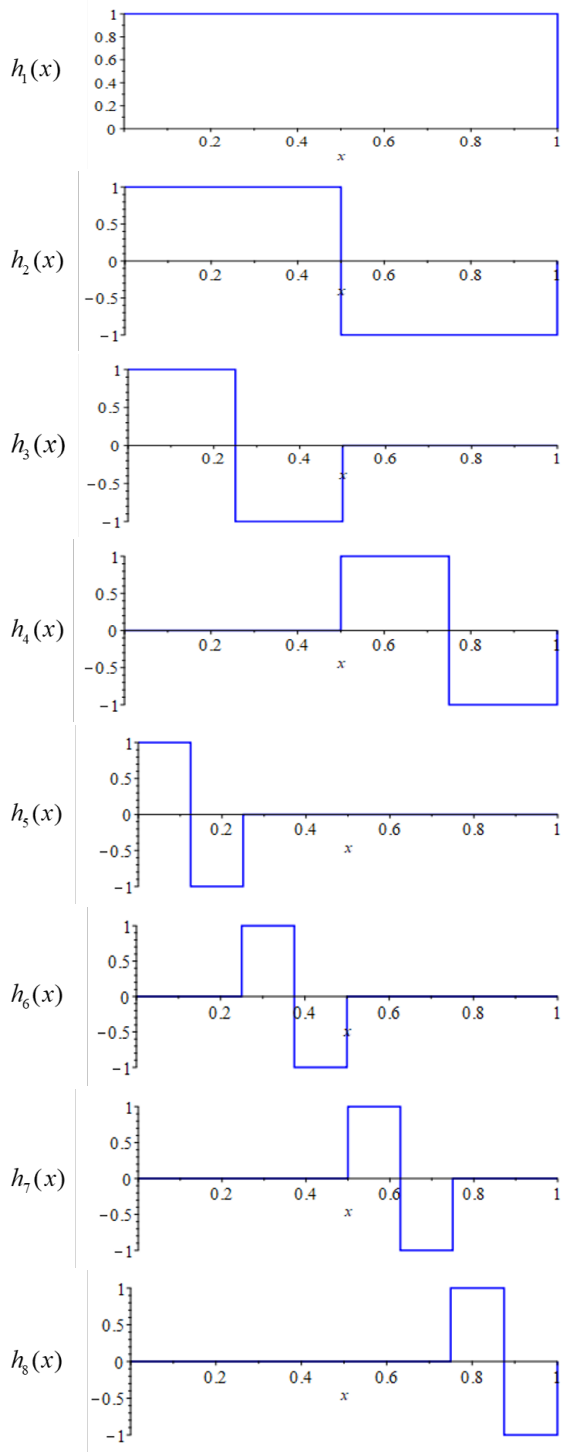

Figure 3. The first eight Haar wavelet functions.

when $i=2^{j}+k, j \geq 0,0 \leq k \leq 2^{j}$ and the Haar coefficients

$$
a_{i}=2^{j} \int_{0}^{1} u(x) h_{i}(x) d x .
$$

In general, the series expansion of $u(x)$ involves infinite terms. Practically, the continuous function $u(x)$ can be approximated by the finite sum of the Haar wavelet, that is

$$
u(x) \approx u_{2 M}(x):=\sum_{i=1}^{2 M} a_{i} h_{i}(x),
$$

where $M=2^{J}$ and the integral square error is defined as

$$
E=\int_{0}^{1}\left[u(x)-u_{2 M}(x)\right]^{2} d x .
$$

The approximation $u_{2 M}(x)$ can be written in the matrix form

$$
u_{2 M}(x)=\mathbf{A}^{T} \mathbf{h},
$$


where $\mathbf{h}=\left[h_{1}(x), h_{2}(x), \ldots, h_{2 M}(x)\right]^{T}$ is the Haar function vector and $\mathbf{A}^{T}=\left[a_{1}, a_{2}, \ldots, a_{2 M}\right]$ is called the coefficient vector. Defining the wavelet collocation points $x_{l}$ as

$$
x_{l}=\frac{l-0.5}{2 M}, \quad l=1,2, \ldots, 2 M .
$$

We construct the Haar wavelet matrix $\mathbf{H}_{2 M}$ of order $2 M$ in which its columns are the Haar function vectors evaluated at $x_{l}, l=1,2, \ldots, 2 M$. In other words, $\mathbf{H}_{2 M}(i, l)=h_{i}\left(x_{l}\right)$, or the $2 M \times 2 M$ matrix $\mathbf{H}_{2 M}$ is

$$
\mathbf{H}_{2 M}=\left[\begin{array}{cccc}
h_{1}\left(x_{1}\right) & h_{1}\left(x_{2}\right) & \ldots & h_{1}\left(x_{2 M}\right) \\
h_{2}\left(x_{1}\right) & h_{2}\left(x_{2}\right) & \ldots & h_{2}\left(x_{2 M}\right) \\
\vdots & \vdots & \ddots & \vdots \\
h_{2 M}\left(x_{1}\right) & h_{2 M}\left(x_{2}\right) & \ldots & h_{2 M}\left(x_{2 M}\right)
\end{array}\right] .
$$

For example, if $J=1 \Rightarrow 2 M=2^{J+1}=4$, then the Haar wavelet matrix of order 4 is

$$
\mathbf{H}_{4 \times 4}=\left[\begin{array}{cccc}
1 & 1 & 1 & 1 \\
1 & 1 & -1 & -1 \\
1 & -1 & 0 & 0 \\
0 & 0 & 1 & -1
\end{array}\right]
$$

and if $J=2 \Rightarrow 2 M=2^{J+1}=8$, then the Haar wavelet matrix of order 8 is

$$
\mathbf{H}_{8 \times 8}=\left[\begin{array}{cccccccc}
1 & 1 & 1 & 1 & 1 & 1 & 1 & 1 \\
1 & 1 & 1 & 1 & -1 & -1 & -1 & -1 \\
1 & 1 & -1 & -1 & 0 & 0 & 0 & 0 \\
0 & 0 & 0 & 0 & 1 & 1 & -1 & -1 \\
1 & -1 & 0 & 0 & 0 & 0 & 0 & 0 \\
0 & 0 & 1 & -1 & 0 & 0 & 0 & 0 \\
0 & 0 & 0 & 0 & 1 & -1 & 0 & 0 \\
0 & 0 & 0 & 0 & 0 & 0 & 1 & -1
\end{array}\right] .
$$

In consequence, we have

$$
\mathbf{U}_{2 M}^{T}=\mathbf{A}^{T} \mathbf{H}_{2 M},
$$

where $\mathbf{U}_{2 M}^{T}=\left[u_{2 M}\left(x_{1}\right), u_{2 M}\left(x_{2}\right), \ldots, u_{2 M}\left(x_{2 M}\right)\right]$ is called the discrete form of the continuous function $u(x)$.

We also concisely provide the basic idea of the integrals of the Haar functions $h_{i}(x)$ of order $n$ denoted by $p_{i, n}(x)$ which can be calculated analytically as follows:

For $i=1$ : the integral of the Haar wavelet, $h_{1}(x)$ of order $n$ is

$$
p_{1, n}(x)=\frac{x^{n}}{n !}, \text { where } n=1,2, \ldots
$$

For $i=2,3, \ldots$ : the integrals of the Haar wavelet $h_{i}(x)$, of the first order $(n=1)$ are [12]

$$
p_{i, 1}(x)=\int_{0}^{x} h_{i}(s) d s= \begin{cases}x-\alpha_{1}, & x \in\left[\alpha_{1}, \alpha_{2}\right), \\ \alpha_{3}-x, & x \in\left[\alpha_{2}, \alpha_{3}\right) \\ 0, & \text { otherwise }\end{cases}
$$

and the integrals of the Haar wavelet, $h_{i}(x)$, order $(n)$ as [20]

$$
\begin{aligned}
& p_{i, n}(x) \\
& =\int_{0}^{x} p_{i, n-1}(s) d s, n=2,3, \ldots \\
& =\frac{1}{n !} \begin{cases}\left(x-\alpha_{1}\right)^{n}, & x \in\left[\alpha_{1}, \alpha_{2}\right), \\
\left(x-\alpha_{1}\right)^{n}-2\left(x-\alpha_{2}\right)^{n}, & x \in\left[\alpha_{2}, \alpha_{3}\right), \\
\left(x-\alpha_{1}\right)^{n}-2\left(x-\alpha_{2}\right)^{n}+\left(x-\alpha_{3}\right)^{n}, & x \in\left[\alpha_{3}, 1\right), \\
0, & \text { otherwise, }\end{cases}
\end{aligned}
$$

where $\alpha_{1}=\frac{k}{m}, \alpha_{2}=\frac{k+0.5}{m}, \alpha_{3}=\frac{k+1}{m}$. Then, we define the $2 M$ operational matric of integrations $\mathbf{P}_{n}$ and its element order $n$, index $i$ is computed using the relation $\mathbf{P}_{n}(i, l)=p_{i, n}\left(x_{l}\right)$, where $x_{l}$ is defined in (17)

For example, if $J=1 \Rightarrow 2 M=4$, and $n=1$, then from (22) the Haar integral matrix of first order and when $n=2$, the Haar wavelet integral matrix of second order is computed by using (23) as

$$
\mathbf{P}_{1}=\frac{1}{8}\left[\begin{array}{cccc}
1 & 3 & 5 & 7 \\
1 & 3 & 3 & 1 \\
1 & 1 & 0 & 0 \\
0 & 0 & 1 & 1
\end{array}\right], \mathbf{P}_{2}=\frac{1}{128}\left[\begin{array}{cccc}
1 & 9 & 25 & 49 \\
1 & 9 & 23 & 31 \\
1 & 7 & 8 & 8 \\
0 & 0 & 1 & 7
\end{array}\right]
$$

For example, if $J=2 \Rightarrow 2 M=8$, and $n=1$, then from (22) we have the Haar integral matrix of first order as

$$
\mathbf{P}_{1}=\frac{1}{16}\left[\begin{array}{cccccccc}
1 & 3 & 5 & 7 & 9 & 11 & 13 & 15 \\
1 & 3 & 5 & 7 & 7 & 5 & 3 & 1 \\
1 & 3 & 3 & 1 & 0 & 0 & 0 & 0 \\
0 & 0 & 0 & 0 & 1 & 3 & 3 & 1 \\
1 & 1 & 0 & 0 & 0 & 0 & 0 & 0 \\
0 & 0 & 1 & 1 & 0 & 0 & 0 & 0 \\
0 & 0 & 0 & 0 & 1 & 1 & 0 & 0 \\
0 & 0 & 0 & 0 & 0 & 0 & 1 & 1
\end{array}\right]
$$

with the graphs as Figure 4.

Considering at $n=2$, the Haar wavelet integral matrix of second order is computed by (23) as

$$
\mathbf{P}_{2}=\frac{1}{512}\left[\begin{array}{cccccccc}
1 & 9 & 25 & 49 & 81 & 121 & 169 & 225 \\
1 & 9 & 25 & 49 & 79 & 103 & 119 & 127 \\
1 & 9 & 23 & 31 & 32 & 32 & 32 & 32 \\
0 & 0 & 0 & 0 & 1 & 9 & 23 & 31 \\
1 & 7 & 8 & 8 & 8 & 8 & 8 & 8 \\
0 & 0 & 1 & 7 & 8 & 8 & 8 & 8 \\
0 & 0 & 0 & 0 & 1 & 7 & 8 & 8 \\
0 & 0 & 0 & 0 & 0 & 0 & 1 & 7
\end{array}\right]
$$

with the graphs as shown in Figure 5. 

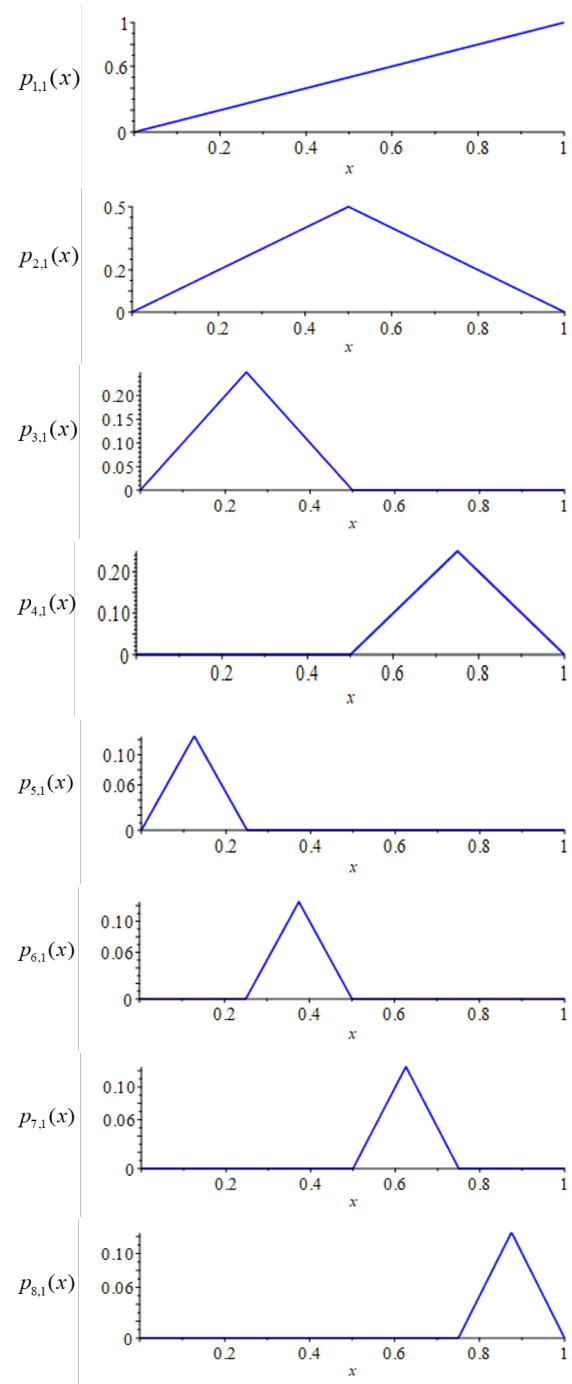

Figure 4. The first eight integrals of Haar wavelet functions $(n=1)$.
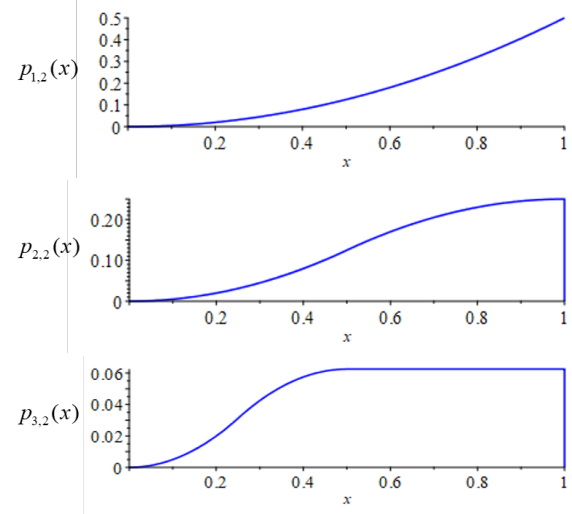

\section{Kronecker product}

Let a matrix $A=\left(a_{i j}\right)$ be dimension $m \times n$ and let a marix $B=\left(b_{i j}\right)$ be dimension $p \times q$ (not necessarily square) as

$A=\left[\begin{array}{cccc}a_{11} & a_{12} & \cdots & a_{1 n} \\ a_{21} & a_{22} & \cdots & a_{2 n} \\ \vdots & \vdots & \ddots & \vdots \\ a_{m 1} & a_{m 2} & \cdots & a_{m n}\end{array}\right], B=\left[\begin{array}{cccc}b_{11} & b_{12} & \cdots & b_{1 q} \\ b_{21} & b_{22} & \cdots & b_{2 q} \\ \vdots & \vdots & \ddots & \vdots \\ b_{p 1} & b_{p 2} & \cdots & b_{p q}\end{array}\right]$
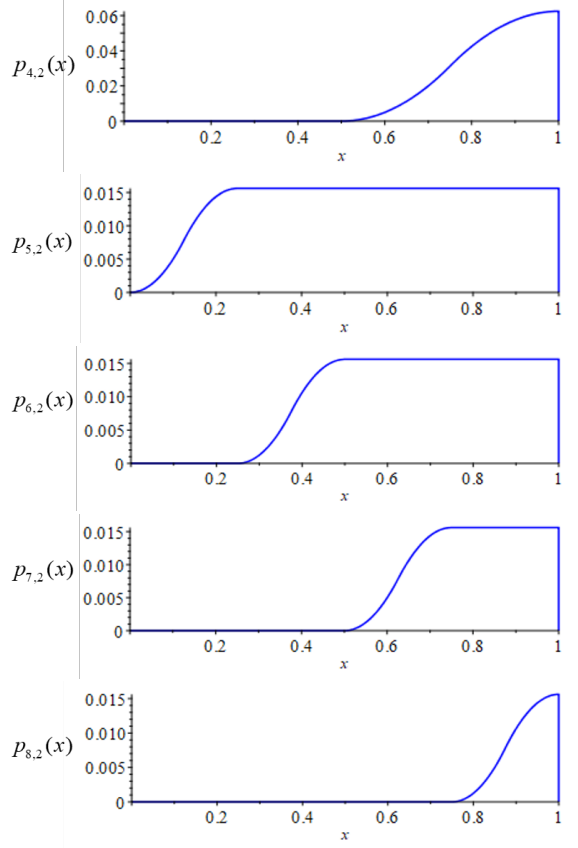

Figure 5. The second eight integrals of Haar wavelet functions $(n=2)$.

The Kronecker product denoted by $A \otimes B$ then it has dimension $(m \times n) \times(p \times q)$ as follows [7]

$$
A \otimes B=\left[\begin{array}{cccc}
a_{11} B & a_{12} B & \cdots & a_{1 n} B \\
a_{21} B & a_{22} B & \cdots & a_{2 n} B \\
\vdots & \vdots & \ddots & \vdots \\
a_{m 1} B & a_{m 2} B & \cdots & a_{m n} B
\end{array}\right],
$$

with some properties

1. $A \otimes(B+C)=(A \otimes B)+(A \otimes C)$,

2. $(A+B) \otimes C=(A \otimes C)+(B \otimes C)$,

3. $A \otimes(k B)=(k A) \otimes B=k(A \otimes B)$,

4. $A \otimes(B \otimes C)=(A \otimes B) \otimes C$,

5. $A \otimes 0=0 \otimes A=0$,

where $A, B$ and $C$ are any matrices, 0 is a zero matrix and $k$ is a scalar. The Kronecker product of matrics $A, B, C$ and $D$ can write the matrix products $A C$ and $B D$ as

$$
(A \otimes B)(C \otimes D)=A C \otimes B D,
$$

which is called the mixed-product property. The accuracy and efficiency of this method had been studied by comparing some HWCM solutions with exact solutions of second order differential equations with several types of boundary conditions [20].

\section{HWCM for a reaction-diffusion equation}

The diffusion equation is a special partial differential equation [2] occur in numerous engineering problems. The simple 
problem requires one initial and two boundary conditions as [10]

$$
\frac{\partial u}{\partial t}=k \frac{\partial^{2} u}{\partial x^{2}}+q(x, t), \quad 0<x<1, t>0,
$$

where $k$ is constant. The initial condition is $u(x, 0)=f(x)$, and boundary conditions are $u(0, t)=g_{0}(t), \frac{\partial u}{\partial x}(1, t)=g_{1}(t)$. Applying HWCM, assuming $u^{(2,1)}(x, t)=\frac{\partial^{3} u}{\partial x^{2} \partial t}$ that expands in terms of two-variable truncated Haar wavelet series can be expressed in the matrix form as

$$
\begin{aligned}
u^{(2,1)}(x, t) & =\sum_{r=1}^{2 M} \sum_{s=1}^{2 M} a_{r, s} h_{r}(x) h_{s}(t), \\
& =H(x) A H(t)
\end{aligned}
$$

where $H(x)=\left[h_{1}(x) h_{2}(x) \ldots h_{2 M}(x)\right]$ and

$$
H(t)=\left[\begin{array}{llll}
h_{1}(t) & h_{2}(t) & \ldots & h_{2 M}(t)
\end{array}\right]^{T} \text { are two vectors of }
$$

Haar functions and $A$ is a coefficient matrix when $N=2 M$ as

$$
A=\left[\begin{array}{cccc}
a_{11} & a_{12} & \cdots & a_{1 N} \\
a_{21} & a_{22} & \cdots & a_{2 N} \\
\vdots & \vdots & & \vdots \\
a_{N 1} & a_{N 2} & \cdots & a_{N N}
\end{array}\right]
$$

Integrating (28) from 0 to $t$ and using the initial condition, we get

$$
u^{(2,0)}(x, t)=H(x) A P_{1}(t)+f^{\prime \prime}(x),
$$

where $P_{1}(t)=\left[p_{1,1}(t) p_{2,1}(t) \ldots p_{2 M, 1}(t)\right]^{T}$. By integrating (28) from 0 to $x$ and the boundary conditions as follows

$$
\begin{aligned}
& u^{(1,1)}(x, t)=P_{1}(x) A H(t)+u^{(1,1)}(0, t), \\
& u^{(0,1)}(x, t)=P_{2}(x) A H(t)+x u^{(1,1)}(0, t)+g_{0}^{\prime}(t),
\end{aligned}
$$

where $P_{1}(x)=\left[p_{1,1}(x) p_{2,1}(x) \ldots p_{2 M, 1}(x)\right]$ and

$$
P_{2}(x)=\left[p_{1,2}(x) p_{2,2}(x) \ldots p_{2 M, 2}(x)\right] \text {. }
$$

Putting $x=1$ into (30), it provides

$$
u^{(1,1)}(0, t)=g_{1}^{\prime}(t)-P_{1}(1) A H(t) .
$$

Substituting (32) into Eqs. (30) and (31), we get

$$
\begin{aligned}
u^{(1,1)}(x, t)= & {\left[P_{1}(x)-P_{1}(1)\right] A H(t)+g_{1}^{\prime}(t) } \\
u^{(0,1)}(x, t)= & {\left[P_{2}(x)-x P_{1}(1)\right] A H(t)+x g_{1}^{\prime}(t) } \\
& +g_{0}^{\prime}(t) .
\end{aligned}
$$

Next, we integrate (29) twice from 0 to $x$ which obtains

$$
\begin{aligned}
u^{(1,0)}(x, t)= & P_{1}(x) A P_{1}(t)+f^{\prime}(x)-f^{\prime}(0) \\
& +u^{(1,0)}(0, t) \\
u^{(0,0)}(x, t)= & P_{2}(x) A P_{1}(t)+f(x)-f(0)-x f^{\prime}(0) \\
& +x u^{(1,0)}(0, t)+g_{0}(t) .
\end{aligned}
$$

Putting $x=1$ into (35), then we have

$$
u^{(1,0)}(0, t)=g_{1}(t)-P_{1}(1) A P_{1}(t)-f^{\prime}(1)+f^{\prime}(0) .
$$

Substituting (37) into Eqs. (35), and (36), we obtain

$$
\begin{aligned}
u^{(1,0)}(x, t)= & {\left[P_{1}(x)-P_{1}(1)\right] A P_{1}(t)+f^{\prime}(x)-f^{\prime}(1) } \\
& +g_{1}(t) .
\end{aligned}
$$

So the HWCM solution is

$$
\begin{aligned}
u^{(0,0)}(x, t)= & {\left[P_{2}(x)-x P_{1}(x)\right] A P_{1}(t)-x\left[g_{1}(t)-f^{\prime}(1)\right.} \\
& \left.+f^{\prime}(0)\right]+f(x)-f(0)+g_{0}(t) .
\end{aligned}
$$

Substituting Eqs. (29) and (34) into (26), the system becomes

$$
\begin{aligned}
{\left[\left(P_{2}(x)\right.\right.} & \left.\left.-x P_{1}(1)\right) A H(t)+x g_{1}^{\prime}(t)+g_{0}^{\prime}(t)\right] \\
& -k\left[H(x) A P_{1}(t)+f^{\prime \prime}(x)\right]-q(x, t)=0 .
\end{aligned}
$$

Considering the Haar wavelet collocation points of space and time are defined by

$$
x_{i}=\frac{i-0.5}{N}, t_{j}=\frac{j-0.5}{N}, \quad i, j=1,2, \ldots, N .
$$

We can generate a system of algebraic equations as

$$
\begin{aligned}
& {\left[\left(P_{2}\left(x_{i}\right)-x_{i} P_{1}(1)\right) A H\left(t_{j}\right)+x_{i} g_{1}^{\prime}\left(t_{j}\right)+g_{0}^{\prime}\left(t_{j}\right)\right]} \\
& \quad-k\left[H\left(x_{i}\right) A P_{1}\left(t_{j}\right)+f^{\prime \prime}\left(x_{i}\right)\right]-q\left(x_{i}, t_{j}\right)=\overline{0} .
\end{aligned}
$$

We also rewrite the (42) into the matrix from as

$$
\begin{aligned}
& {\left[P_{2}^{T}-\left(X \otimes P_{R}\right)\right] A H-k H^{T} A P_{1}} \\
& \quad=Q-X^{T} G_{1}^{\prime}-\Phi\left[G_{0}^{\prime}-F^{\prime \prime}\right]
\end{aligned}
$$

where the matrices are given by

$$
H=\left[\begin{array}{cccc}
h_{1}\left(x_{1}\right) & h_{1}\left(x_{2}\right) & \ldots & h_{1}\left(x_{N}\right) \\
h_{2}\left(x_{1}\right) & h_{2}\left(x_{2}\right) & \ldots & h_{2}\left(x_{N}\right) \\
\vdots & \vdots & \ddots & \vdots \\
h_{N}\left(x_{1}\right) & h_{N}\left(x_{2}\right) & \ldots & h_{N}\left(x_{N}\right)
\end{array}\right]
$$

$$
P_{1}=\left[\begin{array}{cccc}
p_{1,1}\left(x_{1}\right) & p_{1,1}\left(x_{2}\right) & \ldots & p_{1,1}\left(x_{N}\right) \\
p_{2,1}\left(x_{1}\right) & p_{2,1}\left(x_{2}\right) & \ldots & p_{2,1}\left(x_{N}\right) \\
\vdots & \vdots & \ddots & \vdots \\
p_{N, 1}\left(x_{1}\right) & p_{N, 1}\left(x_{2}\right) & \ldots & p_{N, 1}\left(x_{N}\right)
\end{array}\right]
$$

$$
P_{2}=\left[\begin{array}{cccc}
p_{1,2}\left(x_{1}\right) & p_{1,2}\left(x_{2}\right) & \ldots & p_{1,2}\left(x_{N}\right) \\
p_{2,2}\left(x_{1}\right) & p_{2,2}\left(x_{2}\right) & \ldots & p_{2,2}\left(x_{N}\right) \\
\vdots & \vdots & \ddots & \vdots \\
p_{N, 2}\left(x_{1}\right) & p_{N, 2}\left(x_{2}\right) & \ldots & p_{N, 2}\left(x_{N}\right)
\end{array}\right],
$$

$$
\begin{gathered}
X=\left[\begin{array}{llll}
x_{1} & x_{2} & \ldots & x_{N}
\end{array}\right] \\
P_{R}=\left[\begin{array}{llll}
p_{1,1}(1) & p_{2,1}(1) & \ldots & p_{N, 1}(1)
\end{array}\right]^{T},
\end{gathered}
$$

$$
G_{1}^{\prime}=\left[\begin{array}{llll}
g_{1}^{\prime}\left(t_{1}\right) & g_{1}^{\prime}\left(t_{2}\right) & \ldots & g_{1}^{\prime}\left(t_{N}\right)
\end{array}\right],
$$




$$
\begin{gathered}
G_{0}^{\prime}=\left[\begin{array}{llll}
g_{0}^{\prime}\left(t_{1}\right) & g_{0}^{\prime}\left(t_{2}\right) & \ldots & g_{0}^{\prime}\left(t_{N}\right)
\end{array}\right], \\
F^{\prime}=\left[\begin{array}{llll}
f^{\prime}\left(x_{1}\right) & f^{\prime}\left(x_{2}\right) & \ldots & f^{\prime}\left(x_{N}\right)
\end{array}\right], \\
Q=\left[\begin{array}{cccc}
q\left(x_{1}, t_{1}\right) & q\left(x_{1}, t_{2}\right) & \ldots & q\left(x_{1}, t_{N}\right) \\
q\left(x_{2}, t_{1}\right) & q\left(x_{2}, t_{2}\right) & \ldots & q\left(x_{2}, t_{N}\right) \\
\vdots & \vdots & \ddots & \vdots \\
q\left(x_{N}, t_{1}\right) & q\left(x_{N}, t_{2}\right) & \ldots & q\left(x_{N}, t_{N}\right)
\end{array}\right], \\
\Phi=\left[\begin{array}{llll}
1 & 1 & \ldots & 1
\end{array}\right]^{T}
\end{gathered}
$$

and $\overline{0}$ is zero $N \times N$ matrix. We solve (43) with the developing coding HWCM via the Maple program to find the coefficient matrix of the Haar wavelet $A$. After that, we substitute $A$ into (39), and obtain the approximate analytical solution of the diffusion equation (26) with the initial and boundary conditions.

\section{HWCM solutions of the enzymatic glucose fuel cell model}

Consider (5), we have the transport equations for glucose concentration $\left(\left[C_{G}\right]=u\right)$ and hydrogen ion concentration $\left(\left[C_{H^{+}}\right]=v\right)$, when $0<x<1$ and $t \geq 0$ as

$$
\begin{aligned}
\frac{\partial u}{\partial t}= & D_{G} \frac{\partial^{2} u}{\partial x^{2}}-\frac{V_{\max }}{2[E]}\left[(a+u)-\sqrt{(a+u)^{2}-4[E] u}\right] \\
\frac{\partial v}{\partial t}= & D_{H^{+}} \frac{\partial^{2} v}{\partial x^{2}}-y D_{H^{+}} \triangle v^{*} \frac{\partial v}{\partial x} \\
& +\frac{V_{\max }}{[E]}\left[(a+u)-\sqrt{(a+u)^{2}-4[E] u}\right]
\end{aligned}
$$

From Eq. (44), we get

$$
\begin{aligned}
& \frac{2[E]}{V_{\max }}\left(D_{G} \frac{\partial^{2} u}{\partial x^{2}}-\frac{\partial u}{\partial t}\right)=(a+u)-\sqrt{(a+u)^{2}-4[E] u}, \\
& {\left[(a+u)-\frac{2[E]}{V_{\max }}\left(D_{G} \frac{\partial^{2} u}{\partial x^{2}}-\frac{\partial u}{\partial t}\right)\right]^{2}=(a+u)^{2}-4[E] u,}
\end{aligned}
$$

so that

$$
\begin{gathered}
\left(D_{G} \frac{\partial^{2} u}{\partial x^{2}}+\frac{\partial u}{\partial t}\right)^{2}-S\left[\left(D_{G} \frac{\partial^{2} u}{\partial x^{2}}-\frac{\partial u}{\partial t}\right) u\right. \\
\left.+(2 a+u)\left(D_{G} \frac{\partial^{2} u}{\partial x^{2}}-\frac{\partial u}{\partial t}\right)\right]+T u=0 . \\
\left(\frac{\partial v}{\partial t}-D_{H^{+}} \frac{\partial^{2} v}{\partial x^{2}}+C \frac{\partial v}{\partial x}\right)^{2}-2 S\left[\left(\frac{\partial v}{\partial t}-D_{H^{+}} \frac{\partial^{2} v}{\partial x^{2}}+C\right.\right. \\
\left.+(2 a+u)\left(\frac{\partial v}{\partial t}-D_{H^{+}} \frac{\partial^{2} v}{\partial x^{2}}+C \frac{\partial v}{\partial x}\right)\right]+4 T u=0,
\end{gathered}
$$

where $a=[E]+K_{M}, S=\frac{V_{\max }}{2[E]}$ and $T=2 S V_{\max }$. The initial conditions are $u(x, 0)=1, v(x, 0)=0$ and the boundary conditions are $u(0, t)=1, \frac{\partial u}{\partial x}(1, t)=0, v(0, t)=0$ and

$$
\frac{\partial v}{\partial x}(1, t)=0 .
$$

In this example, the collocation points of space $\left(x_{i}\right)$ and time $\left(t_{j}\right)$ when $M=2$ are defined by

$$
x_{i}, t_{j}=\left[\begin{array}{llll}
0.1250 & 0.3750 & 0.6250 & 0.8750
\end{array}\right],
$$

Assuming $\frac{\partial^{3} u}{\partial x^{2} \partial t}\left(x_{i}, t_{j}\right), \frac{\partial^{3} v}{\partial x^{2} \partial t}\left(x_{i}, t_{j}\right)$ can be expressed by the Haar wavelet $(M=2)$ as the matrix form

$$
\begin{aligned}
& \frac{\partial^{3} u}{\partial x^{2} \partial t}\left(x_{i}, t_{j}\right)=H(x) A H(t), \\
& \frac{\partial^{3} v}{\partial x^{2} \partial t}\left(x_{i}, t_{j}\right)=H(x) B H(t),
\end{aligned}
$$

where $A, B$ are coefficient matrices, $N=2 M$, that is

$A=\left[\begin{array}{cccc}a_{11} & a_{12} & \cdots & a_{1 N} \\ a_{21} & a_{22} & \cdots & a_{2 N} \\ \vdots & \vdots & & \vdots \\ a_{N 1} & a_{N 2} & \cdots & a_{N N}\end{array}\right], B=\left[\begin{array}{cccc}b_{11} & b_{12} & \cdots & b_{1 N} \\ b_{21} & b_{22} & \cdots & b_{2 N} \\ \vdots & \vdots & & \vdots \\ b_{N 1} & b_{N 2} & \cdots & b_{N N}\end{array}\right]$

Considering the collocation points (48), we apply (29) with initial condition (6), we get

$\frac{\partial^{2} u}{\partial x^{2}}\left(x_{i}, t_{j}\right)=H_{x} A P_{1}$,

$$
\begin{aligned}
& =\left[\begin{array}{cccc}
1 & 1 & 1 & 1 \\
1 & 1 & -1 & -1 \\
1 & -1 & 0 & 0 \\
0 & 0 & 1 & -1
\end{array}\right]^{T} \times\left[\begin{array}{cccc}
a_{11} & a_{12} & a_{13} & a_{14} \\
a_{21} & a_{22} & a_{23} & a_{24} \\
a_{31} & a_{32} & a_{33} & a_{34} \\
a_{41} & a_{42} & a_{43} & a_{44}
\end{array}\right] \\
& \times\left[\begin{array}{cccc}
0.1250 & 0.3750 & 0.6250 & 0.8750 \\
0.1250 & 0.3750 & 0.3750 & 0.1250 \\
0.1250 & 0.1250 & 0 & 0 \\
0 & 0 & 0.1250 & 0.1250
\end{array}\right],
\end{aligned}
$$

$$
u \frac{\partial^{2} v}{\partial x^{2}}\left(x_{i}, t_{j}\right)=H_{x} B P_{1}
$$

$$
\begin{aligned}
& =\left[\begin{array}{cccc}
1 & 1 & 1 & 1 \\
1 & 1 & -1 & -1 \\
1 & -1 & 0 & 0 \\
0 & 0 & 1 & -1
\end{array}\right]^{T} \times\left[\begin{array}{llll}
b_{11} & b_{12} & b_{13} & b_{14} \\
b_{21} & b_{22} & b_{23} & b_{24} \\
b_{31} & b_{32} & b_{33} & b_{34} \\
b_{41} & b_{42} & b_{43} & b_{44}
\end{array}\right] \\
& \times\left[\begin{array}{cccc}
0.1250 & 0.3750 & 0.6250 & 0.8750 \\
0.1250 & 0.3750 & 0.3750 & 0.1250 \\
0.1250 & 0.1250 & 0 & 0 \\
0 & 0 & 0.1250 & 0.1250
\end{array}\right]
\end{aligned}
$$


From (34) and the boundary conditions (7), obtains

$$
\begin{aligned}
& \frac{\partial u}{\partial t}\left(x_{i}, t_{j}\right)=\left[P_{2}^{T}-\left(x_{i} \otimes P_{1}(1)\right)\right] A H_{t}, \\
& =\left\{\begin{array}{c}
{\left[\begin{array}{cccc}
0.0078 & 0.0703 & 0.1953 & 0.3828 \\
0.0078 & 0.0703 & 0.1797 & 0.2422 \\
0.0078 & 0.0547 & 0.0625 & 0.0625 \\
0 & 0 & 0.0078 & 0.0547
\end{array}\right]^{T}} \\
-\left[\begin{array}{cccc}
0.12375 & 0.37125 & 0.61875 & 0.86625 \\
0.00125 & 0.00375 & 0.00625 & 0.00875 \\
0 & 0 & 0 & 0 \\
0.00125 & 0.00375 & 0.00625 & 0.00875
\end{array}\right]
\end{array}\right\} \\
& \times\left[\begin{array}{llll}
a_{11} & a_{12} & a_{13} & a_{14} \\
a_{21} & a_{22} & a_{23} & a_{24} \\
a_{31} & a_{32} & a_{33} & a_{34} \\
a_{41} & a_{42} & a_{43} & a_{44}
\end{array}\right] \times\left[\begin{array}{cccc}
1 & 1 & 1 & 1 \\
1 & 1 & -1 & -1 \\
1 & -1 & 0 & 0 \\
0 & 0 & 1 & -1
\end{array}\right] \\
& =\left[\begin{array}{cccc}
-0.11595 & -0.36345 & -0.61095 & -0.86625 \\
0.06905 & 0.06655 & 0.04845 & -0.00875 \\
0.19530 & 0.17970 & 0.06250 & 0.00780 \\
0.38155 & 0.23845 & 0.05625 & 0.04595
\end{array}\right] \\
& \times\left[\begin{array}{llll}
a_{11} & a_{12} & a_{13} & a_{14} \\
a_{21} & a_{22} & a_{23} & a_{24} \\
a_{31} & a_{32} & a_{33} & a_{34} \\
a_{41} & a_{42} & a_{43} & a_{44}
\end{array}\right] \times\left[\begin{array}{cccc}
1 & 1 & 1 & 1 \\
1 & 1 & -1 & -1 \\
1 & -1 & 0 & 0 \\
0 & 0 & 1 & -1
\end{array}\right],
\end{aligned}
$$

From (38) and the boundary conditions (7), obtains

$$
\begin{aligned}
& \frac{\partial v}{\partial x}\left(x_{i}, t_{j}\right)=\left[P_{1}^{T}-P_{1}^{T}(1)\right] B P_{1} \\
& =\left\{\begin{array}{c}
{\left[\begin{array}{cccc}
0.1250 & 0.3750 & 0.6250 & 0.8750 \\
0.1250 & 0.3750 & 0.3750 & 0.1250 \\
0.1250 & 0.1250 & 0 & 0 \\
0 & 0 & 0.1250 & 0.1250
\end{array}\right]^{T}} \\
-\left[\begin{array}{cccc}
0.99 & 0.99 & 0.99 & 0.99 \\
0.01 & 0.01 & 0.01 & 0.01 \\
0 & 0 & 0 & 0 \\
0.01 & 0.01 & 0.01 & 0.01
\end{array}\right]^{T}
\end{array}\right\} \\
& \times\left[\begin{array}{llll}
b_{11} & b_{12} & b_{13} & b_{14} \\
b_{21} & b_{22} & b_{23} & b_{24} \\
b_{31} & b_{32} & b_{33} & b_{34} \\
b_{41} & b_{42} & b_{43} & b_{44}
\end{array}\right] \\
& \times\left[\begin{array}{cccc}
0.1250 & 0.3750 & 0.6250 & 0.8750 \\
0.1250 & 0.3750 & 0.3750 & 0.1250 \\
0.1250 & 0.1250 & 0 & 0 \\
0 & 0 & 0.1250 & 0.1250
\end{array}\right] \\
& =\left[\begin{array}{cccc}
-0.8650 & -0.6150 & -0.3650 & -0.1150 \\
0.1150 & 0.3650 & 0.3650 & 0.1150 \\
0.1250 & 0.1250 & 0 & 0 \\
-0.0100 & -0.0100 & 0.1150 & 0.1150
\end{array}\right] \\
& \times\left[\begin{array}{llll}
b_{11} & b_{12} & b_{13} & b_{14} \\
b_{21} & b_{22} & b_{23} & b_{24} \\
b_{31} & b_{32} & b_{33} & b_{34} \\
b_{41} & b_{42} & b_{43} & b_{44}
\end{array}\right] \\
& \times\left[\begin{array}{cccc}
0.1250 & 0.3750 & 0.6250 & 0.8750 \\
0.1250 & 0.3750 & 0.3750 & 0.1250 \\
0.1250 & 0.1250 & 0 & 0 \\
0 & 0 & 0.1250 & 0.1250
\end{array}\right]
\end{aligned}
$$

From (39) and the boundary conditions (7), obtains

$$
\begin{aligned}
& \frac{\partial v}{\partial t}\left(x_{i}, t_{j}\right)=\left[P_{2}^{T}-\left(x_{i} \otimes P_{1}(1)\right)\right] B H_{t}, \\
& =\left\{\begin{array}{c}
{\left[\begin{array}{cccc}
0.0078 & 0.0703 & 0.1953 & 0.3828 \\
0.0078 & 0.0703 & 0.1797 & 0.2422 \\
0.0078 & 0.0547 & 0.0625 & 0.0625 \\
0 & 0 & 0.0078 & 0.0547
\end{array}\right]^{T}} \\
-\left[\begin{array}{cccc}
0.12375 & 0.37125 & 0.61875 & 0.86625 \\
0.00125 & 0.00375 & 0.00625 & 0.00875 \\
0 & 0 & 0 & 0 \\
0.00125 & 0.00375 & 0.00625 & 0.00875
\end{array}\right]
\end{array}\right\} \\
& \times\left[\begin{array}{llll}
b_{11} & b_{12} & b_{13} & b_{14} \\
b_{21} & b_{22} & b_{23} & b_{24} \\
b_{31} & b_{32} & b_{33} & b_{34} \\
b_{41} & b_{42} & b_{43} & b_{44}
\end{array}\right] \times\left[\begin{array}{cccc}
1 & 1 & 1 & 1 \\
1 & 1 & -1 & -1 \\
1 & -1 & 0 & 0 \\
0 & 0 & 1 & -1
\end{array}\right] \\
& =\left[\begin{array}{cccc}
-0.11595 & -0.36345 & -0.61095 & -0.86625 \\
0.06905 & 0.06655 & 0.04845 & -0.00875 \\
0.19530 & 0.17970 & 0.06250 & 0.00780 \\
0.38155 & 0.23845 & 0.05625 & 0.04595
\end{array}\right] \\
& \times\left[\begin{array}{llll}
b_{11} & b_{12} & b_{13} & b_{14} \\
b_{21} & b_{22} & b_{23} & b_{24} \\
b_{31} & b_{32} & b_{33} & b_{34} \\
b_{41} & b_{42} & b_{43} & b_{44}
\end{array}\right] \times\left[\begin{array}{cccc}
1 & 1 & 1 & 1 \\
1 & 1 & -1 & -1 \\
1 & -1 & 0 & 0 \\
0 & 0 & 1 & -1
\end{array}\right]
\end{aligned}
$$




$$
\begin{aligned}
u\left(x_{i}, t_{j}\right)= & {\left[P_{2}^{T}-\left(x_{i} \otimes P_{1}(1)\right)\right] A P_{1}+g_{0}\left(t_{j}\right) } \\
= & {\left[\begin{array}{cccc}
-0.11595 & -0.36345 & -0.61095 & -0.86625 \\
0.06905 & 0.06655 & 0.04845 & -0.00875 \\
0.19530 & 0.17970 & 0.06250 & 0.00780 \\
0.38155 & 0.23845 & 0.05625 & 0.04595
\end{array}\right] } \\
& \times\left[\begin{array}{cccc}
a_{11} & a_{12} & a_{13} & a_{14} \\
a_{21} & a_{22} & a_{23} & a_{24} \\
a_{31} & a_{32} & a_{33} & a_{34} \\
a_{41} & a_{42} & a_{43} & a_{44}
\end{array}\right] \\
\times & {\left[\begin{array}{cccc}
0.1250 & 0.3750 & 0.6250 & 0.8750 \\
0.1250 & 0.3750 & 0.3750 & 0.1250 \\
0.1250 & 0.1250 & 0 & 0 \\
0 & 0 & 0.1250 & 0.1250
\end{array}\right] } \\
+ & {\left[\begin{array}{cccc}
1 & 1 & 1 & 1 \\
1 & 1 & 1 & 1 \\
1 & 1 & 1 & 1 \\
1 & 1 & 1 & 1
\end{array}\right] }
\end{aligned}
$$

and

$$
\begin{aligned}
v\left(x_{i}, t_{j}\right)= & {\left[P_{2}-\left(x_{i} \otimes P_{1}^{T}(1)\right)\right] B P_{1} } \\
= & {\left[\begin{array}{cccc}
-0.11595 & -0.36345 & -0.61095 & -0.86625 \\
0.06905 & 0.06655 & 0.04845 & -0.00875 \\
0.19530 & 0.17970 & 0.06250 & 0.00780 \\
0.38155 & 0.23845 & 0.05625 & 0.04595
\end{array}\right] } \\
& \times\left[\begin{array}{cccc}
b_{11} & b_{12} & b_{13} & b_{14} \\
b_{21} & b_{22} & b_{23} & b_{24} \\
b_{31} & b_{32} & b_{33} & b_{34} \\
b_{41} & b_{42} & b_{43} & b_{44}
\end{array}\right] \\
& \times\left[\begin{array}{cccc}
0.1250 & 0.3750 & 0.6250 & 0.8750 \\
0.1250 & 0.3750 & 0.3750 & 0.1250 \\
0.1250 & 0.1250 & 0 & 0 \\
0 & 0 & 0.1250 & 0.1250
\end{array}\right]
\end{aligned}
$$

Substituting Eqs. (51), (53) and (56) into (46) and Eqs. (52), (54), (55) and (57) into (47), we then develop the HWCM coding in the Maple program to find the coefficient matrices of Haar wavelet $A$ and $B$. We obtain

$$
\begin{aligned}
A & =\left[\begin{array}{cccc}
-0.0755 & -0.0081 & 0.0835 & -0.0075 \\
-0.2709 & 0.0997 & 0.1713 & -0.1125 \\
-0.8613 & -0.2198 & 1.0811 & -0.0001 \\
0.1954 & -0.1077 & -0.0878 & 0.1050
\end{array}\right], \\
B & =\left[\begin{array}{cccc}
0.0566 & 0.0473 & -0.1039 & -0.0111 \\
0.2452 & 0.1124 & -0.3577 & 0.2474 \\
0.6031 & 0.5976 & -1.2001 & -0.4136 \\
-0.1887 & -0.0651 & 0.2538 & -0.2585
\end{array}\right]
\end{aligned}
$$

Thus, the HWCM solution of the glucose concentration is given by

$$
\begin{aligned}
u(x, t)= & {\left[P_{2}(x)-x P_{1}(x)\right] } \\
& \times\left[\begin{array}{cccc}
-0.0755 & -0.0081 & 0.0835 & -0.0075 \\
-0.2709 & 0.0997 & 0.1713 & -0.1125 \\
-0.8613 & -0.2198 & 1.0811 & -0.0001 \\
0.1954 & -0.1077 & -0.0878 & 0.1050
\end{array}\right] P_{1}(t) \\
& +g_{0}(t)
\end{aligned}
$$

and the HWCM solution of the hydrogen ion concentration is given by

$$
\begin{aligned}
v(x, t)= & {\left[P_{2}(x)-x P_{1}(x)\right] } \\
& \times\left[\begin{array}{cccc}
0.0566 & 0.0473 & -0.1039 & -0.0111 \\
0.2452 & 0.1124 & -0.3577 & 0.2474 \\
0.6031 & 0.5976 & -1.2001 & -0.4136 \\
-0.1887 & -0.0651 & 0.2538 & -0.2585
\end{array}\right] P_{1}(t) .
\end{aligned}
$$

\section{Numerical Results}

Some numerical results for the glucose and hydrogen ion concentrations in the enzymatic glucose fuel cell model can be shown as the approximate analytical solutions. All solutions are computed by the Haar wavelet collocation method (HWCM) with coding $(M=2)$ in the Maple program with the parameters values in Table 1.

Figure 6 shows that the glucose concentration decreases along with a distance $(x)$ and increasing time $(t)$. Initially, glucose enters the cell with full constant concentration, $1 \mathrm{~mol} \cdot \mathrm{m}^{-3}$ at $t=0$. After a while, the glucose concentration decreases exponentially. The hydrogen ion concentration increases across the cell. At the beginning of time $(t)$ hydrogen ion concentration rapidly increases.

Table 1. Value of Parameters (estimation).

\begin{tabular}{ccc}
\hline Parameters & Values & Units \\
\hline$C_{G}$ & & $\mathrm{~mol} \cdot \mathrm{m}^{-3}$ \\
$C_{H^{+}}$ & & $\mathrm{mol} \cdot \mathrm{m}^{-3}$ \\
$D_{G}$ & 0.2 & $\mathrm{~m}^{2} \mathrm{~s}^{-1}$ \\
$D_{H+}$ & 0.5 & $\mathrm{~m}^{2} \mathrm{~s}^{-1}$ \\
{$[E]$} & 1 & $\mathrm{~mol} \cdot \mathrm{m}^{-2}$ \\
$K_{c a t}$ & 0.5 & $\mathrm{~s}^{-1}$ \\
$V_{\text {max }}$ & 0.5 & $\mathrm{~mol} \cdot \mathrm{m}^{-2} \mathrm{~s}^{-1}$ \\
$K_{M}$ & 2 & $\mathrm{~mol} \cdot \mathrm{m}^{-3}$ \\
$\triangle v^{*}$ & 0.1 & $\mathrm{~V}$ \\
$L$ & 1 & $\mathrm{~m}^{-3}$ \\
$y$ & 2 & - \\
\hline
\end{tabular}

The glucose and hydrogen ion concentrations with various glucose diffusion coefficient, $D_{G}=0.2,0.5,1,2$, and hydrogen ion diffusion coefficient, $D_{H^{+}}=0.5,1.2,1.5,4$, can be shown in Figure 7 .

\section{Conclusions}

This paper proposes the enzymatic glucose fuel cell model by applying enzyme kinetics, the Morrison equation. The approximate analytical solutions for glucose and hydrogen ion concentrations in enzymatic glucose fuel cell can be solved by the Haar wavelet collocation method (HWCM). It is obvious that glucose concentration decreases across the cell. Moreover, glucose concentration rapidly decreases when diffusion coefficients of glucose decrease, but the hydrogen ion concentration increases when the diffusion coefficients of hydrogen ion decrease.

\section{Acknowledgements}

This research was financially supported by King Mongkut's University of Technology North Bangkok, Thailand under contract no. 

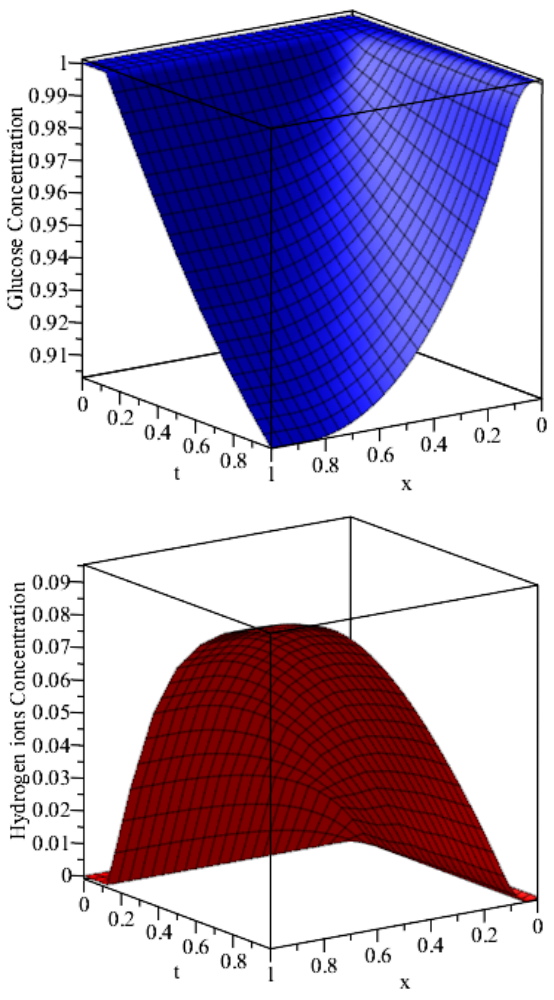

Figure 6. Graphs of glucose and hydrogen ion concentrations by the HWCM at $0<x<1$ and $0<t<1$.
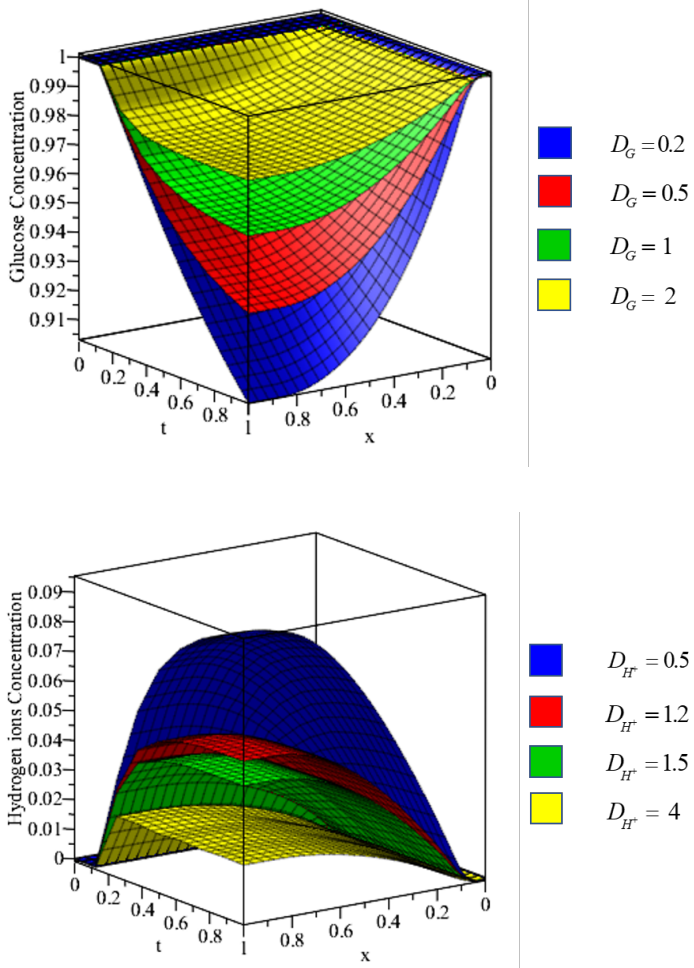

Figure 7. Compare graphs of the glucose and hydrogen ion concentrations diffusion coefficient of glucose and hydrogen ion at $0<x<1$ and $0<t<$ 1.

KMUTNB-64-NEW-14, and partially supported by the Centre of Excellence in Mathematics, the Commission on Higher Education, Thai- land.

\section{REFERENCES}

[1] A. Meena, A. Eswari, and L. Rajendran, "Mathematical modelling of enzyme kinetics reaction mechanisms and analytical solutions of non-linear reaction equations," Journal of mathematical chemistry, vol. 48, no. 2, pp. 179-186, 2010.

[2] B. Abraham-Shrauner, "Exact traveling wave solutions of nonlinear evolution equations: Indeterminant homogeneous balance and linearizability," Mathematics and Statistics, pp. 10-13, 2019.

[3] C. Chen and C. Hsiao, "Haar wavelet method for solving lumped and distributed-parameter systems," IEE Proceedings-Control Theory and Applications, vol. 144, no. 1, pp. 87-94, 1997.

[4] D. Schultz, V. Shah, W. Shay, and P. Wang, "Diffusion of oxygen and carbon dioxide through blood flowing in a channel," Medical and Biological Engineering and Computing, vol. 15, no. 2, pp. 98-105, 1977.

[5] E. Hetmaniok, K. Kaczmarek, D. Słota, R. Wituła, and A. Zielonka, "Application of the variational iteration method for determining the temperature in the heterogeneous casting-mould system,” Int. Rev. Chem. Eng, vol. 4, no. 5, 2012.

[6] F. A. Shah and R. Abass, "An operational Haar wavelet collocation method for solving singularly perturbed boundary-value problems," SeMA Journal, vol. 74, no. 4, pp. 457-474, 2017.

[7] F. J. Caro-Lopera, V. Leiva, and N. Balakrishnan, "Connection between the Hadamard and matrix products with an application to matrix-variate Birnbaum-Baunders distributions," Journal of Multivariate Analysis, vol. 104, pp. 126-139, 2012.

[8] G. Hariharan and K. Kannan, "Review of wavelet methods for the solution of reaction-diffusion problems in science and engineering," Applied Mathematical Modelling, vol. 38, no. 3, pp. 799-813, 2014.

[9] 'I. Çelik, "Chebyshev wavelet collocation method for solving generalized Burgers-Huxley equation," Mathematical methods in the applied sciences, vol. 39, no. 3, pp. 366-377, 2016.

[10] I. Youssef and A. Ali, "Memory effects in diffusion like equation via Haar wavelets," Pure and Applied Mathematics Journal, vol. 5, no. 4, pp. 130-140, 2016.

[11] J. Morrison, "Kinetics of the reversible inhibition of enzymecatalysed reactions by tight-binding inhibitors," Biochimica et Biophysica Acta (BBA)-Enzymology, vol. 185, no. 2, pp. 269-286, 1969.

[12] J. Majak, M. Pohlak, K. Karjust, M. Eerme, J. Kurnitski, and B. Shvartsman, "New higher order Haar wavelet method: Application to fgm structures," Composite Structures, vol. 201, pp. 72-78, 2018.

[13] L. Bougoffa, "The Adomian decomposition method for solving a moving boundary problem arising from the diffusion of oxygen in absorbing tissue," The Scientific World Journal, vol. 2014, 2014.

[14] L. del Torno-de Román, M. Navarro, G. Hughes, J. P. Esquivel, R. D. Milton, S. D. Minteer, and N. Sabaté, "Improved performance of a paper-based glucose fuel cell by capillary induced flow," Electrochimica Acta, vol. 282, pp. 336-342, 2018. 
[15] L. Rajendran, M. Kirthiga, and E. Laborda, "Mathematical modeling of nonlinear reaction-diffusion processes in enzymatic biofuel cells," Current Opinion in Electrochemistry, vol. 1, no. 1, pp. 121-132, 2017.

[16] M. Goličnik, "Evaluation of enzyme kinetic parameters using explicit analytic approximations to the solution of the michaelis-menten equation,” Biochemical Engineering Journal, vol. 53, no. 2, pp. 234-238, 2011.

[17] M. Ramanathan, R. Muthuramalingam, and R. Lakshmanan, "The mathematical theory of diffusion and reaction in enzymes immoblized artificial membrane the theory of the non-steady state," The Journal of membrane biology, vol. 248, no. 6, pp. 1127-1135, 2015.

[18] M. Razzaghi and S. Yousefi, "The Legendre wavelets operational matrix of integration," International Journal of Systems Science, vol. 32, no. 4, pp. 495-502, 2001.

[19] O. González-Gaxiola, "The Laplace-Adomian decomposition method applied to the Kundu-Eckhaus equation," arXiv preprint arXiv:1704.07730, 2017
[20] R. Yangchareonyuanyong, S. Koonprasert, and S. Sirisubtawee, "Numerical solutions of the elliptic differential and the planetary motion equations by haar wavelet-quasilinearization technique," Thai Journal of Mathematics, pp. 351-370, 2020.

[21] S. C. Barton, "1d models for enzymatic biological fuel cells," The Electrochemical Society Interface, vol. 24, no. 3, pp. 61-65, 2015.

[22] S. Kumar and R. Singh, "Solution of reaction-diffusion equations by using homotopy perturbation method," International Journal of Stability and Fluid Mechanics, vol. 1, no. 2, pp. 235-244, 2010

[23] S. Jariwala, S. Phul, R. Nagpal, S. Goel, and B. Krishnamurthy, "Modeling the performance of enzymatic glucose fuel cells," Journal of Electroanalytical Chemistry, vol. 801, pp. 354-359, 2017.

[24] Ü. Lepik, "Numerical solution of differential equations using Haar wavelets," Mathematics and computers in simulation, vol. 68, no. 2, pp. 127-143, 2005. 